Conclusion. We have observed clear differences in the pattern of referrals made to the adult PLNS during the first COVID-19 national lockdown. COVID-19 was implicated in a minority of referrals, but most were related to secondary effects of lockdown restrictions rather than COVID-19 infection. Possible reasons for fewer referrals during this time could be non-presentation through fears of contracting COVID-19 or altruistic avoidance of putting "pressure on the NHS". Further studies would be insightful; in particular, equivalent analysis of contacts with community services; and qualitative patient perspectives regarding reasons for non-presentation during this time.

\section{Longterm cognitive dysfunction in paediatric brain tumour survivors - the need for multifactorial risk screening}

\author{
Ashy Rengit \\ Kent \& Medway NHS Partnership Trust
}

doi: 10.1192/bjo.2021.174

Aims. Identify common risk factors for longterm cognitive dysfunction in PBTS (paediatric brain tumour survivors) Examine how various paediatric cancer treatment modalities affect cognitive outcomes Consider baseline features which may increase the risk of cognitive dysfunction in PBTS

Method. Current research into the neuropsychiatric sequelae of childhood brain tumours is limited, therefore review of the literature was conducted to identify research within this field.

Databases

Google Scholar - papers accessed via the University of Brighton or Sussex online library

NICE HDAS - HMIC, AMED, MEDLINE, BNI, PsycINFO, CINAHL, Pubmed, EMBASE \& EMCARE

Mendeley reference manager - papers for background reading

Search terms

PICO(T) method - Population (Cancer Survivors), Intervention (Cancer Treatment), Comparison (Brain tumour), Outcome (Cognitive dysfunction) \& Time (Childhood \& adolescence) Boolean operators (AND/OR), truncation and wildcard search functions were also utilised.

Inclusion criteria; no limits on date, study type or gender, however, study results were limited by age - as the research focus was restricted to children and adolescents.

Excluded results; papers which did not meet inclusion criteria, duplicate studies, studies measuring non-cognitive cancer outcomes or investigating non-cortical tumours, non-English language studies with no available English translations.

Result. Common risk factors - certain tumour types (glioneuronal tumours or gliomas) or inner cortical tumour sites e.g. were more vulnerable to epileptogenesis. In particular, seizures which were prolonged and treatment-resistant were associated with a greater degree of cognitive dysfunction.

Impact of various cancer treatment modalities - overall results understandably suggested that patients are more likely to develop cognitive deficits following brain tumour treatment. In particular, partial tumour resection (especially if epileptogenic), whole-brain irradiation, cranial radiotherapy and chemotherapy were more likely to impact cognitive function.

Baseline features that may increase likelihood of cognitive dysfunction e.g. intellectual disability or education level were not noted in the reviewed literature.

Conclusion. Cancer is one of the leading causes of global child mortality, and younger populations often present to paediatric oncology services with brain tumour involvement. Current childhood brain tumour research has begun to recognise that many young survivors develop into adulthood with cognitive sequelae impacting quality of life measures. However, existing evidence is also limited and requires further research to produce a standardised clinical tool for screening various risk factors which may increase longterm risk of cognitive dysfunction and subsequent difficulties with daily life.

\section{Sexual and reproductive health needs assessment \& interventions in a female psychiatric intensive care} unit

Elizabeth Rose ${ }^{1 \star}$, Elana Covshoff ${ }^{2}$, Lucy Blake ${ }^{1}$, Adenike Bolade ${ }^{1}$, Robert Rathouse $^{1}$, Aleshia Wilson ${ }^{1}$, Arthur Cotterell ${ }^{1}$, Rudiger Pittrof ${ }^{2}$ and Faisil Sethi ${ }^{3}$

${ }^{1}$ South London and Maudsley NHS Foundation Trust; ${ }^{2}$ Guy's and St Thomas NHS Foundation Trust and ${ }^{3}$ Dorset Healthcare University NHS Foundation Trust

${ }^{*}$ Corresponding author.

doi: 10.1192/bjo.2021.175

Aims. To assess the sexual and reproductive health (SRH) needs of women admitted to a psychiatric intensive care unit (PICU), and acceptability of delivering specialist SRH assessments/interventions in this setting. Secondary aims were to explore the barriers to access and the feasibility of providing SRH assessments and interventions in the PICU.

Method. A retrospective analysis of fifteen months' activity data found that only 25 SRH referrals had been made across 205 PICU admissions. This low referral rate of $12 \%$ likely reflected pathway barriers and was unlikely to represent the actual clinical need in female PICU patients. A bi-monthly SRH in-reach clinic and a nurse led SRH referral pathway were implemented on the PICU over a seven-month period. Within a quality improvement framework, a staff training needs assessment was performed, training delivered, a protocol developed, staff attitudes explored, and patient and carer engagement sought.

Result. A quality improvement approach streamlined SRH assessments on the PICU and resulted in $42 \%$ of women being assessed and a 3.5 -fold increase in uptake. At least $30 \%$ of the women in the PICU had unmet SRH needs identified and proceeded to a specialist appointment. This amounts to a minimum 2.5-fold increase in SRH unmet need detection.

The most common SRH needs were complex gynaecological issues (such as period problems, pelvic pain, vaginal discharge), STI advice/testing and contraception advice/options. $21 \%$ of women initiated SRH interventions, and $14 \%$ completed all the interventions required for their needs. The most common interventions were in the areas of contraception advice/family planning and STI advice/testing.

Staff confidence on assessing SRH topics was identified as a barrier to access with a positive shift noted after bespoke SRH training was implemented and a protocol introduced: on a scale of 0-10 (with 10 being high), $81.3 \%$ of staff rated their confidence 8 or above in relation to discussing contraception/sexually transmitted infections (pre-training: $25.0 \%$ ), and $93.8 \%$ in relation to discussing risky behaviours (pre-training: 18.8\%). All 11 patient and carer participants felt it was important to have a forum to talk about SRH and $8(72.7 \%)$ agreed it was important in the PICU.

Conclusion. Results identify that SRH needs for PICU admissions are greater than previously realised. Staff highlighted the acceptability and importance of SRH care, if interventions are appropriately timed and the patient's individual risk profile considered. 